AperTO - Archivio Istituzionale Open Access dell'Università di Torino

\title{
Combining drug-loaded nanobubbles and Extracorporeal Shock Waves for difficult-to-treat cancers
}

This is a pre print version of the following article:

Original Citation:

Availability:

This version is available http://hdl.handle.net/2318/1662346

since 2018-03-14T15:14:23Z

Published version:

DOI: $10.2174 / 1567201814666171018120430$

Terms of use:

Open Access

Anyone can freely access the full text of works made available as "Open Access". Works made available under a Creative Commons license can be used according to the terms and conditions of said license. Use of all other works requires consent of the right holder (author or publisher) if not exempted from copyright protection by the applicable law. 


\section{IIIS AperTO}

UNIVERSITÀ

DEGLI STUDI

DI TORINO

This is the author's final version of the contribution published as:

Roberta Cavalli, Francesca Marano, Monica Argenziano, Alessandra Varese, Roberto Frairia and Maria Graziella Catalano

Combining Drug-Loaded Nanobubbles and Extracorporeal Shock Waves for Difficult-to-Treat Cancers

Current Drug Delivery, 2017, vol. 14

DOI: $10.2174 / 1567201814666171018120430$

The publisher's version is available at:

http://www.eurekaselect.com/156461/article

When citing, please refer to the published version.

Link to this full text:

http://hdl.handle.net/hdl:2318/1662346 


\section{Combining drug-loaded nanobubbles and Extracorporeal Shock Waves for difficult-to-treat cancers}

Despite the general great improvement in cancer therapy, to date, some aggressive tumors are still without an efficient therapy. Therefore, accurate delivery of anti-cancer drugs is a very important goal in order to obtain a successful therapy and reduce systemic side effects.

Nanobubbles (NBs) are spherical core/shell vesicles filled by a gas with sizes in the nanometer order of magnitude. They have gained an increasing attention for drug delivery, because they can be versatile multifunctional carriers for the targeted release of gases, drugs and genes. Particularly, NBs can carry loaded drugs to the tumor site through the blood stream, taking advantage of the enhanced permeability and retention effect, due to the defective vascular architecture of the tumor (Fang et al. 2011). Unfortunately, vessel leakage, the absence of a functional lymphatic system and an increased extracellular matrix frictional resistance may limit drug delivery (Azzi et al. 2013, Carmeliet and Jain 2011). To overcome this problem, a better drug release to cancer tissues can be obtained by combining physical triggers (e.g. ultrasounds, US) with NBs (Gao et al. 2008, Collis et al. 2010, Cavalli et al. 2012, Cavalli et al. 2016). Indeed, US causes bubble cavitation resulting in cell sonoporation and allowing the extravasation of molecules (Collis et al. 2010).

Interestingly, polysaccharide-shelled perfloropentane (PFP)-cored NBs were developed for the delivery of anticancer agents displaying good drug encapsulation efficiency and a prolonged release kinetics. This type of formulation is referred to as "nanobubbles" for sake of simplicity but it must be said that, prior to the application of external stimuli such as US, it would be more accurate to use the term "nanodroplets" when the core is constituted of PFP, being a perfluorocarbon liquid at room temperature (boiling point $29^{\circ} \mathrm{C}$ ) (Cavalli et al. 2015).

Extracorporeal Shock Waves (ESWs), introduced in medicine about 40 years ago (Eisenberger et al. 1978), are short-duration $(<10 \mu \mathrm{s})$ focused acoustic waves consisting of a compressive phase (peak pressure: 10-100 MPa) followed by a tensile phase (negative pressure). ESW therapy is widely used in urology for lithotripsy to break urinary stones (Rassweiler et al. 2011). In addition to 
lithotripsy, more recently, ESWs have been successfully used for the treatment of several musculoskeletal diseases (Wang et al. 2012). ESWs can be focused with high precision also in depth and they determine permeabilization of plasma membranes (Frairia et al. 2003, Catalano et al. 2007), as a consequence of cavitation occurring when shockwaves propagate into fluids. As result of induced cell permeability, ESWs increase the cytotoxic effects of different anti-cancer drugs (Frairia et al. 2003, Palmero et al. 2006, Catalano et al. 2007, Canaparo et al. 2008). Notably, ESWs differ from US as they have 1000 times higher peak pressure (Wang et al. 2012), and no heating effects. The absence of heating effect is an advantage for in vivo application since temperature elevation is difficult to control spatially and temporally, especially in large tumors with heterogeneous vascularization (Diederich et al. 1999). All these features make ESWs an ideal tool to be used in combination with drug-loaded NBs. Indeed, drug-loaded NBs can be injected intravenously and, when they have reached the tumor, ESWs can be applied directly at the tumor site. At this level, in fact, ESW-induced cavitation might determine perturbation of the NBs in addition to transient permeabilization of both plasma and nuclear membranes, resulting in the drug release and penetration into the cells.

The effects of combining chemotherapeutic drug-loaded NBs and ESWs have been recently investigated into two different types of aggressive cancers; e.g., anaplastic thyroid cancer (ATC) and castration resistant prostate cancer (CRPC).

In ATC, we obtained both in vitro and in vivo data. In vitro, the combined treatment with doxorubicin-loaded glycol chitosan NBs and ESWs enhanced cytotoxicity of doxorubicin in two different ATC cells lines (CAL-62 and 8305C), decreasing the drug growth inhibition fifty (GI ${ }_{50}$ ) of 40\% in CAL-62 and 46\% in 8305C cells, respectively (Marano et al. 2016). The reduction of the $\mathrm{GI}_{50}$ is notable, since the use of lower doses of doxorubicin is essential to reduce its side effects. In a xenograft in vivo model, after 21 days, the combined treatment determined the greatest drug accumulation in tumors with consequent reduction of tumor volume and weight, and an extension of the tumor doubling time. Mechanistically, the treatment induced tumor apoptosis and decreased 
cell proliferation. Finally, loading doxorubicin into NBs avoided and prevented heart damage, the most frequent side effect of the free drug that causes heart fibrosis and oxidative stress (Marano et al. 2017).

In CRPC, Marano et al. (2016) reported that the combined treatment with either paclitaxel- or docetaxel-loaded NBs and ESWs enhanced the cytotoxicity of both taxanes in two different cell lines (PC3 and DU145), resulting in paclitaxel $\mathrm{GI}_{50}$ reduction of about 55\% and in docetaxel $\mathrm{GI}_{50}$ reduction of about 45\%, respectively. Moreover, the proposed combination therapy was effective also on cell migration. Paclitaxel-loaded NBs and ESWs reduced cell migration by more than $85 \%$ with respect to paclitaxel alone; whereas docetaxel-loaded NBs and ESWs reduced cell migration by more than $82 \%$ with respect to docetaxel alone.

Given the promising results of these preclinical studies, combining drug-loaded NBs and ESWs should be taken into account also for other aggressive tumors in which chemotherapy remains the first option. The next step will be conducting clinical trials with the hope of improving cancer treatment as well as patient quality of life. 


\section{References}

Azzi S, Hebda JK, Gavard J. Vascular permeability and drug delivery in cancers. Front Oncol. 2013; 3:211.

Canaparo R, Serpe L, Zara GP, Chiarle R, Berta L, Frairia R. High energy shock waves (ESW) increase paclitaxel efficacy in a syngeneic model of breast cancer. Technol Cancer Res Treat. 2008; 7(2):117-124.

Carmeliet P, Jain RK. Principles and mechanisms of vessel normalization for cancer and other angiogenic diseases. Nat Rev Drug Discov. 2011;10(6):417-427.

Catalano MG, Costantino L, Fortunati N, Bosco O, Pugliese M, Boccuzzi G, Berta L, Frairia R. High energy shock waves activate 5'-aminolevulinic Acid and increase permeability to Paclitaxel: antitumor effects of a new combined treatment on anaplastic thyroid cancer cells. Thyroid. 2007; 17(2):91-99.

Cavalli R, Bisazza A, Trotta M, Argenziano M, Civra A, Donalisio M, Lembo D. New chitosan nanobubbles for ultrasound-mediated gene delivery: preparation and in vitro characterization. Int $\mathrm{J}$ Nanomedicine. 2012; 7:3309-3318.

Cavalli R, Argenziano M, Vigna E, Giustetto P, Torres E, Aime S, Terreno E. Preparation and in vitro characterization of chitosan nanobubbles as theranostic agents. Colloids Surf B Biointerfaces. 2015;129:39-46.

Cavalli R, Soster M, Argenziano M. Nanobubbles: a promising efficient tool for therapeutic delivery. Ther Deliv. 2016;7(2):117-38. doi: 10.4155/tde.15.92. Epub 2016 Jan 15. Review. PubMed PMID: 26769397.

Collis J, Manasseh R, Liovic P, Tho P, Ooi A, Petkovic-Duran K, Zhu Y Cavitation microstreaming and stress fields created by microbubbles. Ultrasonics. 2010; 50(2):273-279.

Diederich CJ, Hynynen K. Ultrasound technology for hyperthermia. Ultrasound Med Biol. 1999; 25(6):871-887. 
Eisenberger F, Chaussy C. Contact-free renal stone fragmentation with shock waves. Urol Res. 1978; 6(3):111.

Fang J, Nakamura H, Maeda H. The EPR effect: Unique features of tumor blood vessels for drug delivery, factors involved, and limitations and augmentation of the effect. Adv Drug Deliv Rev. 2011; 63(3):136-151

Frairia R, Catalano MG, Fortunati N, Fazzari A, Raineri M, Berta L. High energy shock waves (HESW) enhance paclitaxel cytotoxicity in MCF-7 cells. Breast Cancer Res Treat. 2003; 81(1):1119.

Gao Z, Kennedy AM, Christensen DA, Rapoport NY. Drug-Loaded Nano/Microbubbles for Combining Ultrasonography and Targeted Chemotherapy. Ultrasonics. 2008; 48(4):260-270.

Marano F, Argenziano M, Frairia R, Adamini A, Bosco O, Rinella L, Fortunati N, Cavalli R, Catalano MG. Doxorubicin-Loaded Nanobubbles Combined with Extracorporeal Shock Waves: Basis for a New Drug Delivery Tool in Anaplastic Thyroid Cancer. Thyroid. 2016 May;26(5):705 16. doi: 10.1089/thy.2015.0342. Epub 2016 Mar 17. PubMed PMID: 26906083.

Marano F, Frairia R, Rinella L, Argenziano M, Bussolati B, Grange C, Mastrocola R, Castellano I, Berta L, Cavalli R, Catalano MG. Combining doxorubicin-nanobubbles and shockwaves for anaplastic thyroid cancer treatment: preclinical study in a xenograft mouse model. Endocr Relat Cancer. 2017 Jun;24(6):275-286. doi: 10.1530/ERC-17-0045. Epub 2017 May 9. PubMed PMID:28487350.

Marano F, Rinella L, Argenziano M, Cavalli R, Sassi F, D'Amelio P, Battaglia A, Gontero P, Bosco O, Peluso R, Fortunati N, Frairia R, Catalano MG. Targeting Taxanes to Castration-Resistant Prostate Cancer Cells by Nanobubbles and Extracorporeal Shock Waves. PLoS One. 2016 Dec 21;11(12):e0168553. doi: 10.1371/journal.pone.0168553. eCollection 2016. PubMed PMID: 28002459; PubMed Central PMCID: PMC5176187.

Palmero A, Berger M, Venturi C, Ferrero I, Rustichelli D, Berta L, Frairia R, Madon E, Fagioli F High energy shock waves enhance the cytotoxic effect of doxorubicin and methotrexate to human osteosarcoma cell lines. Oncol Rep. 2006; 15(1):267-273. 
Rassweiler JJ, Knoll T, Kohrmann KU, McAteer JA, Lingeman JE, Cleveland RO, Bailey MR, Chaussy C. Shock wave technology and application: an update. Eur Urol. 2011; 59(5):784-796.

Wang CJ. Extracorporeal shockwave therapy in musculoskeletal disorders. J Orthop Surg Res. 2012; 7:11. 